\title{
A striped pattern of snowfall and snow cover
}

\author{
YASUAKI NOHgUchi, TAKASHI IKaRASHI, \\ Nagaoka Institute of Snow and Ice Studies, NIED, Suyoshi, Nagaoka, Niigata 940, Japan \\ Osamu Abe and Atsushi Sato \\ Shinjo Branch of Snow and Ice Studies, NIED, Shinjo, Yamagata 996, Japan
}

\begin{abstract}
A striped pattern can be seen by spraying ink on a vertical wall of a snow pit to observe the layered structure of a snow cover. This pattern is caused by variations of snowfall in time, particularly pauses in snowfall, and its structure is related to a kind of fractal. In this paper, we consider snowfall and snow cover from a viewpoint of fractals and show that the layered structure of snow cover is a record of fractals on atmospheric-turbulence phenomena through the time variation of snowfall.
\end{abstract}

\section{INTRODUCTION}

In snow-pit observations, we usually describe the snow cover as a set of finite, countable snow layers. However, when we spray blue ink diluted with water on the vertical wall of the snow pit, we can see a striped pattern composed of numerous blue lines, which looks as if it were endlessly repeated to a finer structure. As a result, descriptions of layers in a snow cover might be strongly observer-dependent.

In general, this striped pattern is caused by the discontinuity of grain-size of snow particles (Wakahama, 1963), and the discontinuity is due to variations of snowfall in time. Each blue line of the striped pattern usually corresponds to a boundary between successive snowfalls (Shimizu, 1965), in other words, a pause in snowfall.

On the other hand, Kawakami and Yoshida (1988) have shown that the variation in precipitation can be considered as a kind of fractal (Mandelbrot, 1981) which is characterized by endlessly repeated geometrical figures, that is, self-similarity or self-affinity. From the viewpoint of fractals, a set of precipitation events on the time axis can be regarded as a cantor set, which is a typical example of fractals. A cantor set is composed of points embedded in space with topological dimension 1 and has an infinite number of pauses in several sizes.

The shapes of clouds are well-known fractals (Lovejoy, 1982) related to atmospheric-turbulence phenomena. Precipitation also is a kind of atmospheric-turbulence phenomenon; therefore, it is suggested that the self-similar structure of the record of precipitation is caused by atmospheric-turbulence phenomena.

Snow is deposited on the ground, in a time-dependent manner, while rain flows away from the ground. Therefore, such a structure of snowfall must be recorded within the snow cover. This paper describes the endlessly repeated structure of the striped pattern in natural snow cover as a kind of record of a fractal of the time variation in snowfall, in which atmospheric-turbulence phenomena are reflected. The purpose of this paper is to inspect the number of snow layers contained in a snow cover and the total time of snowfall through a qualitative model from the viewpoint of fractals.

\section{NUMBER OF SNOW LAYERS}

Figure 1 shows an example of a striped pattern on the vertical wall of a snow pit dyed by spraying blue ink diluted with water. This striped pattern is composed of numerous blue lines in several thicknesses and darknesses. The snow cover can be roughly separated into several layers by the thickest and darkest lines, and each layer also has several thinner lines. Moreover, if we observe carefully the layers between the thinner lines, several further thinner lines can be found within each layer.

Such a striped pattern of snow cover has a layered structure endlessly repeated to a finer structure, as ideally schematized in Figure 2. A snow cover, then, can be considered as a set of uncountable and infinite layers with infinitesimal thickness; actually it is impossible to recognize the layers thinner than the grain-size of snow particles.

\section{TOTAL TIME OF SNOWFALL}

Figure 3 is an example of a record of daily new-snow depth measured by a snow plate in Nagaoka, Japan, in the winter 1985-86. In this record, the time axis is covered with either snowfall periods or its pauses, and a successive snowfall period corresponds to a severe storm, which usually occurs several times per winter in Nagaoka. On the other hand, the small graph included in Figure 3 shows time variation in precipitation, magnifying the 


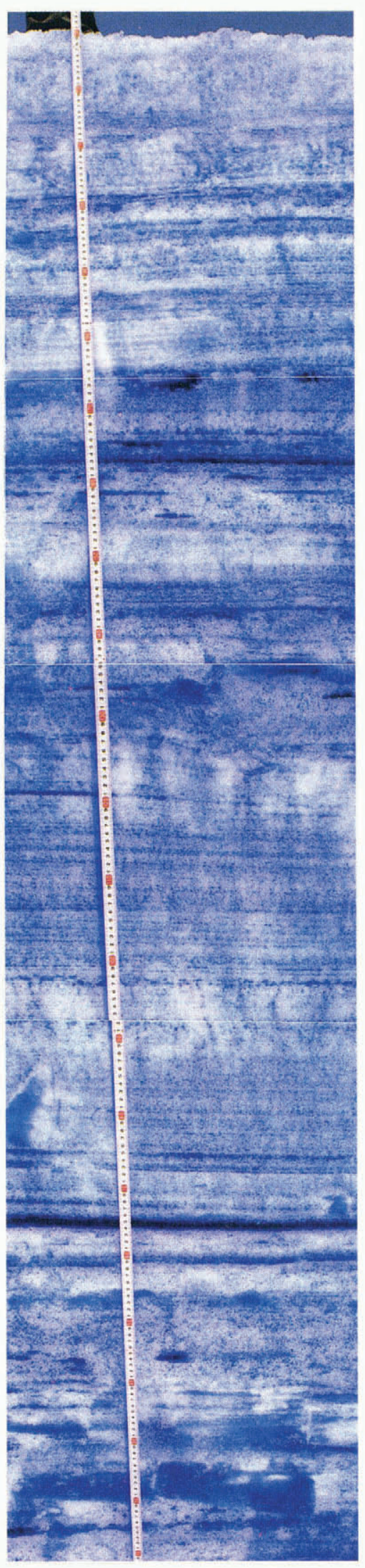

Fig. 1. Striped pattern of snow cover in a pit wall.

time scale of a period during successive snowfalls in the large graph of daily new-snow depth. From this, it can be found that pauses of minimum time scale one hour exist in the record, even though the snowfall is recorded as continuous.

This means that whether or not the snowfall is regarded as continuous depends on the minimum time scale; so does the total time of snowfall. For example, even

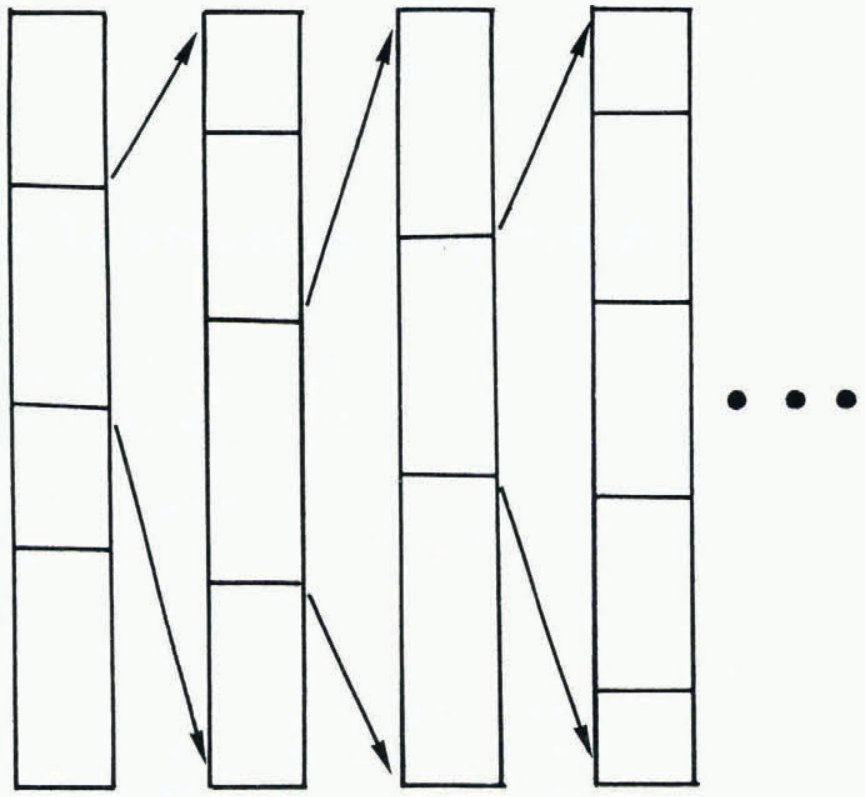

Fig. 2. Schematic representation of a striped pattern of snow cover.

though its total time in a record (minimum time scale $1 \mathrm{~d}$ ) is $10 \mathrm{~d}$, this does not mean that the total time is $240 \mathrm{~h}$ when the minimum time is $1 \mathrm{~h}$. As the snowfall time in the limit of microscopic scales, consider the time for which ice of snow flakes passes through a point near the ground. Assuming that total ice thickness in one wintertime is $1 \mathrm{~m}$ and falling velocity of snow is $1 \mathrm{~m} \mathrm{~s}^{-1}$, snowfall time per one wintertime can be estimated to be $1 \mathrm{~s}$. In this microscopic scale, snowfall time is negligibly small in comparison with total length of a winter, which is about $10^{7} \mathrm{~s}$, even though it snows every day.

\section{SNOW LAYERS AS REFLECTION OF SNOWFALL}

Figure 4 shows schematically the relation between snowfalls and the striped pattern of a snow cover. In general, the striped pattern on the wall of a snow pit is caused by discontinuity of grain-size of snow particles (Wakahama, 1963), and each line of the striped pattern corresponds to each position where vertical distribution of its grain-size changes discontinuously. Usually, each

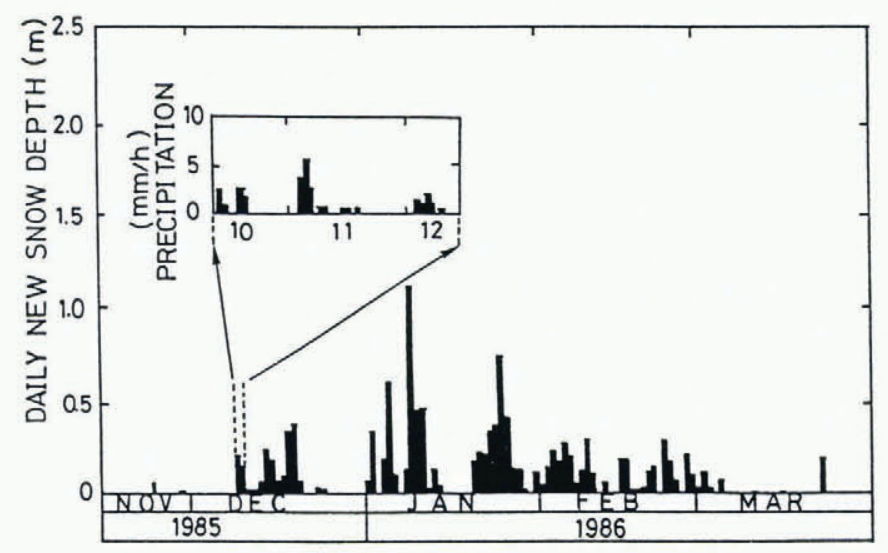

Fig. 3. Daily new-snow depth and precipitation in Nagaoka, Japan, in the 1985-86 winter. 


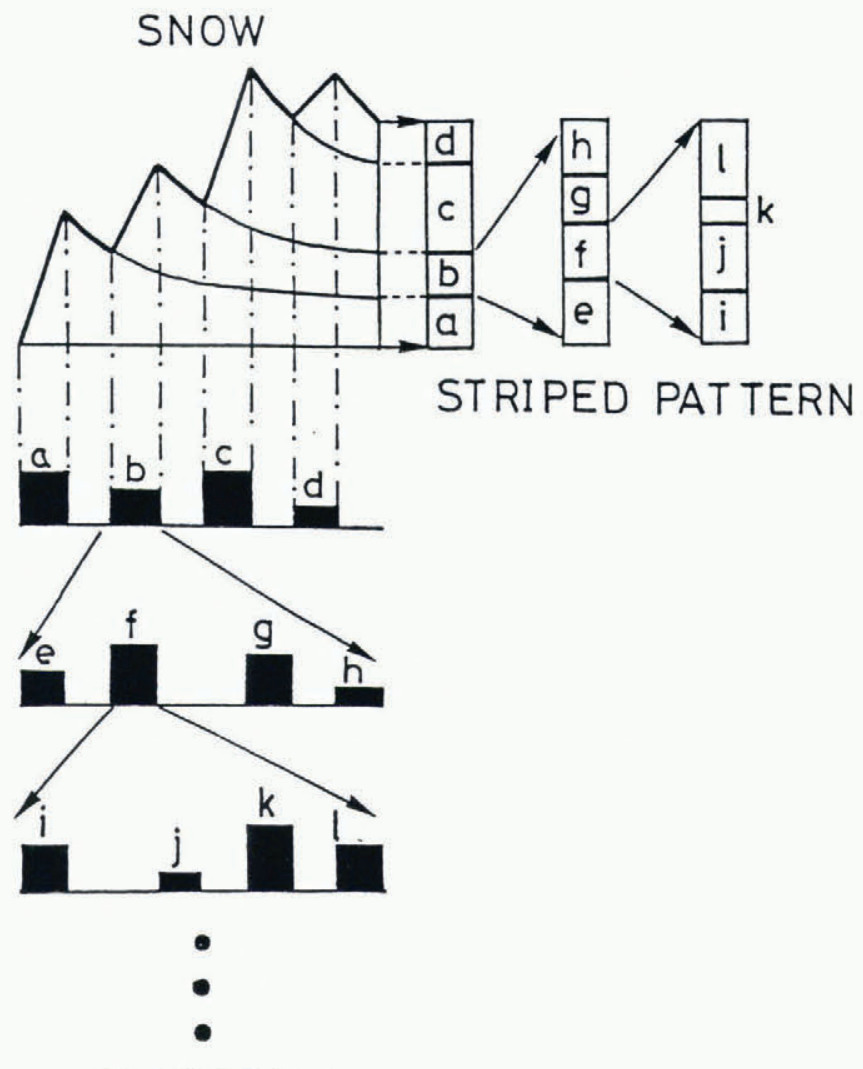

\section{SNOWFALL}

Fig. 4. Schematic representation of successive snowfalls and a striped pattern.

position of such discontinuity coincides with each boundary between a successive snowfall and the following successive one (Shimizu, 1965), as long as it is not disturbed by rainfall or erosion by wind.

Therefore, if snowfall has some characteristic structures on its time variation, then the snow cover made of the snowfall also has some characteristics reflecting the structures of the snowfall through the striped pattern of the snow cover.

\section{DEVIL'S STAIRCASE}
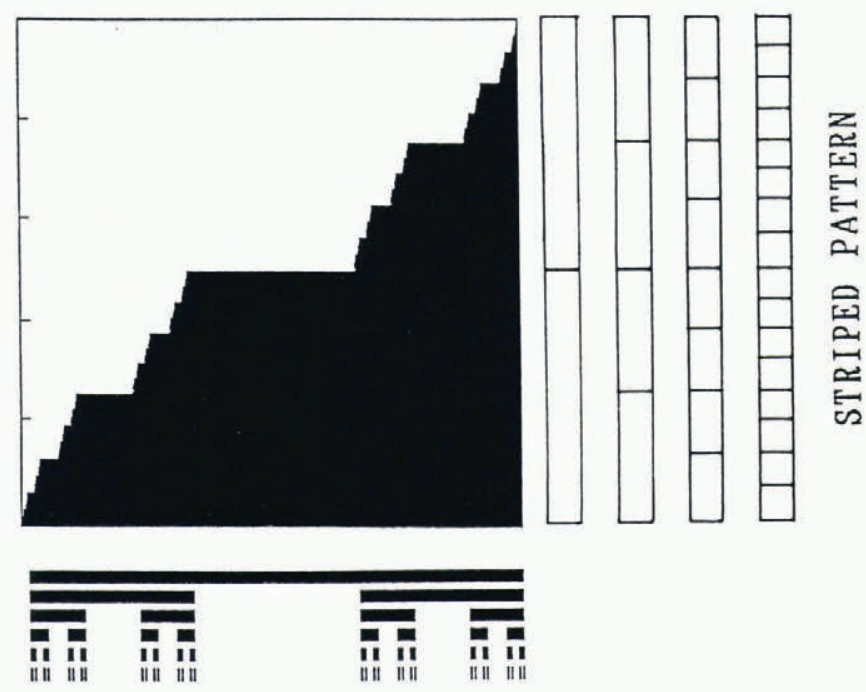

A CANTOR SET

Fig. 5. A cantor set, devil's staircase and striped pattern.
QUALITATIVE MODEL FOR SNOWFALL AND SNOW LAYERS IN A VIEWPOINT OF FRACTAL

A shape made of parts similar in some way to the whole is called a fractal (Mandelbrot, 1986) and it can be characterized by fractal dimension which is not integer. A cantor set (Figure 5) is a typical example of fractals embedded in space with topological dimension 1, and given as the limit of the repeat of the operation as shown in Figure 5. Therefore, a cantor set is composed of an infinite number of gaps. As a result, the total length of every point belonging to the set is zero. If we consider a cantor set as a qualitative model for snowfall, this corresponds to the fact that the total snowfall time in the limit of the microscopic time scale is negligibly small, as mentioned above.

Consider a density-distribution function in which a constant weight is equally distributed on each point belonging to the cantor set. The function which integrates the density-distribution function is called a devil's staircase. If we consider the cantor set as a kind of snowfall record on the time axis, then the devil's staircase corresponds to the records of the snow cover, e.g. time variation in snow load or accumulated daily new-snow depth.

If settlement of snow due to gravity can be neglected, the devil's staircase can be considered as a snow depth. Then, a striped pattern of virtual snow cover made of the cantor set is shown on the right side of the devil's staircase in Figure 5. In this striped pattern, each line is drawn at the depth corresponding to each landing of the devil's staircase. In Figure 5, only a few lines are shown, but ideally the number of lines is infinite, and the virtual snow cover must be perfectly covered with the infinite lines.

Figure 6 is an example of snowfall records observed in

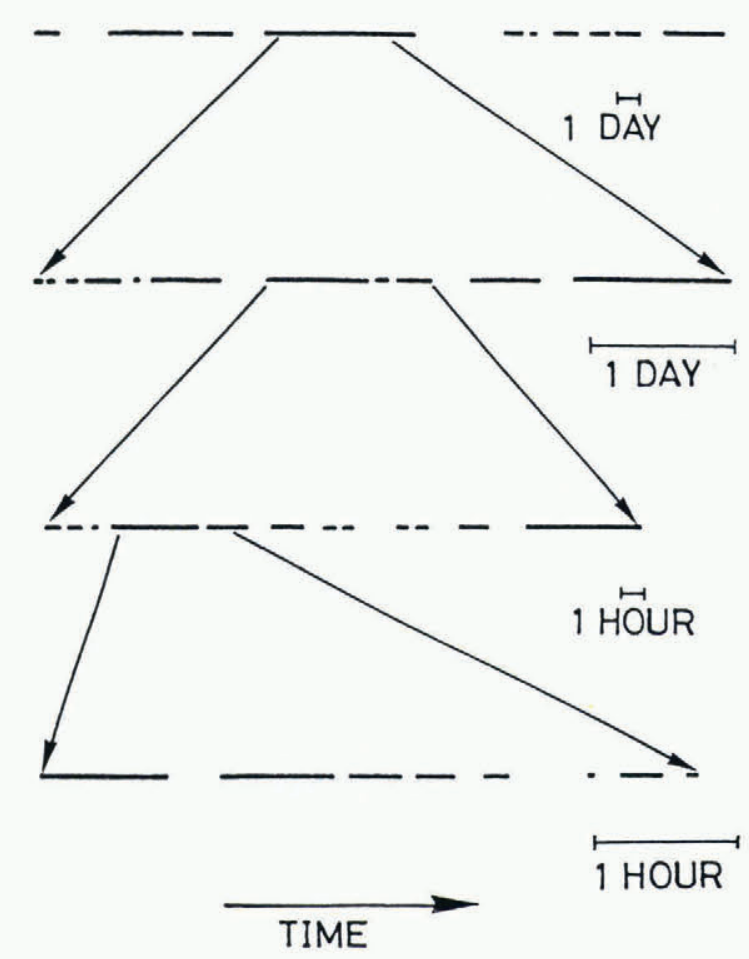

Fig. 6. Snowfall record by a snowfall detector in Koide, Japan, in the 1979-80 winter. Solid lines and gaps represent snowfall period and its pause, respectively. 


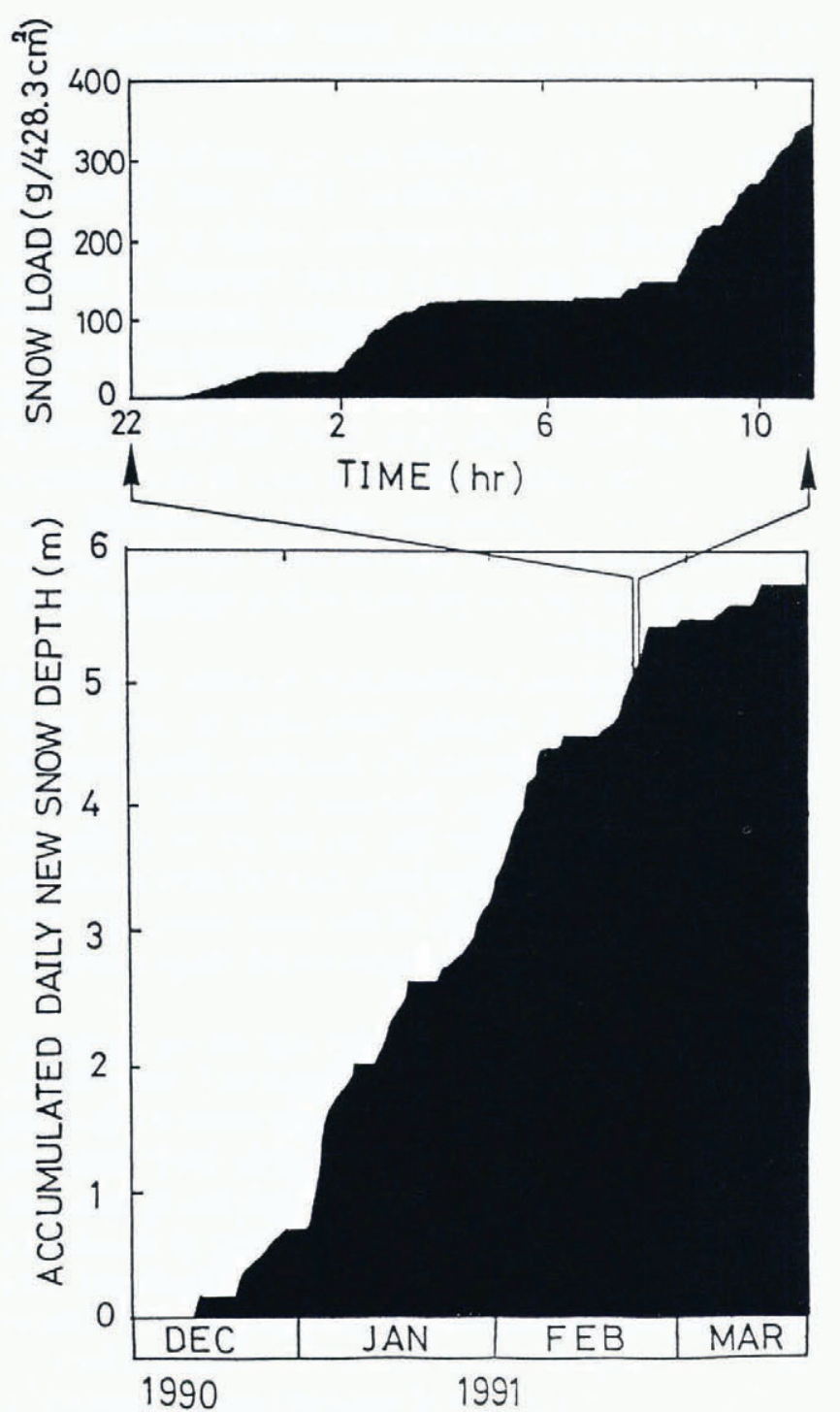

Fig. 7. Devil's staircase by accumulated daily new-snow depth $(\mathrm{m})$ and snow load $\left(\mathrm{g} \mathrm{cm}^{-2}\right)$ in Shinjo, Japan, in the 1990-91 winter.

Koide, Japan, in the winter of $1979-80$ by a snowfall detector, in which the presence of snowfall is detected by the change of the electric resistance at an inclined hot plate. In Figure 6, magnifying the minimum time scale to recognize snowfall, successive snowfall periods are magnified. This shows that the snowfall record on time axis includes a lot of gaps, and that snowfall is not successive at all, even though it looks successive on the larger time scale. Consequently, from this viewpoint, the record of snowfall phenomena on the time axis can be considered as a kind of cantor set.

Figure 7 is an example of actual devil's staircases made of accumulated daily new-snow depth in Shinjo, Japan, in the 1990-91 winter (after Shinjo Branch of Snow and Ice Studies, NIED) and accumulated weight of snowfall measured by an electric balance; the area of the collecting plate is $428 \mathrm{~cm}^{2}(25.8 \mathrm{~cm} \times 16.6 \mathrm{~cm})$. In the record measured by the electric balance, its time scale is spread in comparison with that in the record of accumulated daily new-snow depth. In Figure 7, we can see many landings with several scales in time.

From the viewpoint of fractals, the number of snow layers contained in a snow cover is dependent on scale and becomes infinite in the limit of microscale. This is consistent with the fact that the total time of snowfall also depends on scale and becomes zero in the limit of microscale. Therefore, it does not make sense to obtain the total time of snowfall or the number of snow layers absolutely, i.e. independently of scale.

\section{FRAGTAL DIMENSION OF SNOWFALL}

We obtain a fractal dimension for snowfall from a set of snowfall times by using a box-counting method (Feder, 1988). In this method, the objective period is divided into small sections $\Delta$ in length, and the number $N(\Delta)$ of the sections in which snowfall is included is counted. If the snowfall is successive without pause and can be considered as a continuous segment on the time axis, then the total time of snowfall is

$$
T(\Delta)=\Delta \times N(\Delta) .
$$

It is independent of the time scale $\Delta$, and is constant. As mentioned above, however, the total of snowfall time $T$ depends in general on the time scale $\Delta$ and is not constant.

When the number $N$ can be represented by the following power function

$$
N(\Delta)=c \times \Delta^{-D},
$$

this index $D$ is called the fractal dimension. As a particular case, when $D=1, T$ becomes constant. This

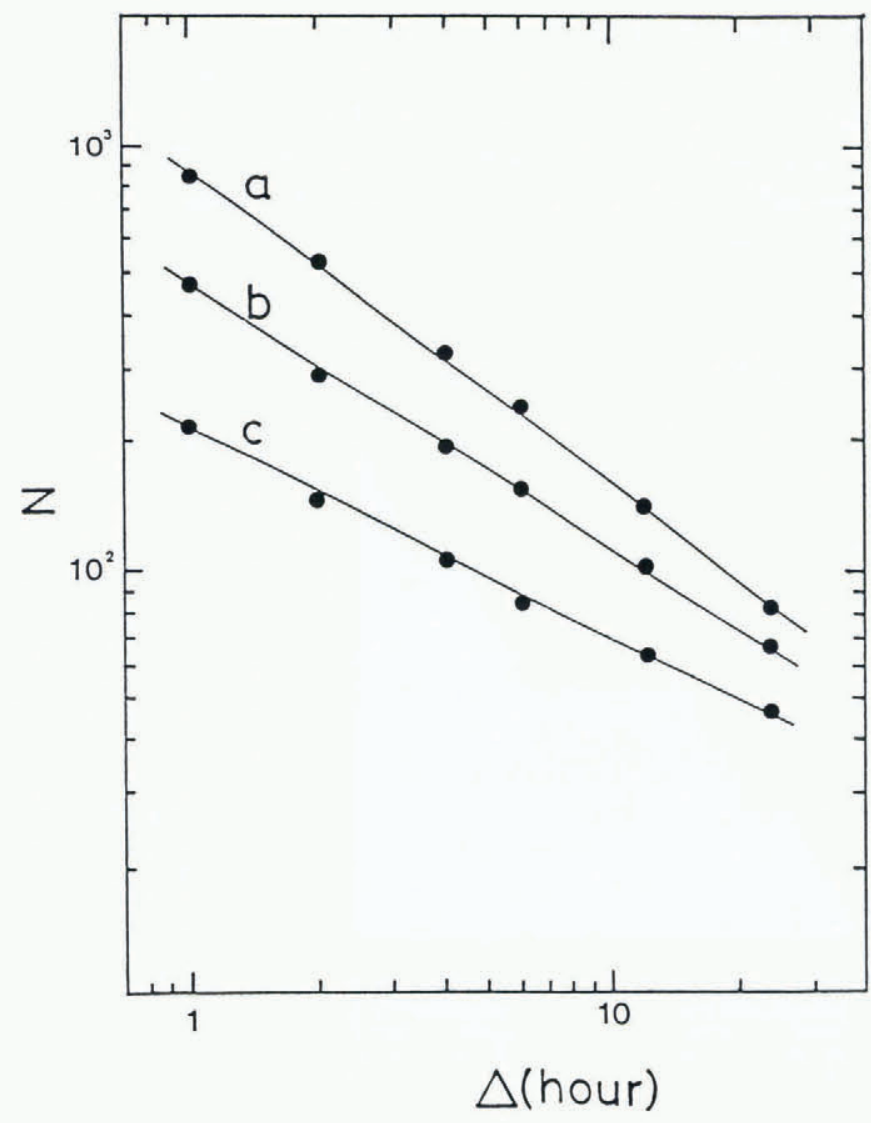

Fig. 8. Log-log plots of precipitation in Nagaoka, Japan, in the winter of December 1985-March 1986. 
Nohguchi and others: A striped pattern of snowfall and snow cover

indicates that snowfall is one-dimensional if it is continuous, therefore it coincides with the concept of dimension which we usually use. In this method, the fractal dimension is obtained from a slope of the straight line drawn by the log-log plots of $N$ and $\Delta$.

Figure 8 shows an example of these log-log plots of the records of precipitation measured by an overflow type rain/snowgauge in the winter of December 1985-March 1986 in Nagaoka. In this analysis, only precipitation which is equal to or more than a threshold value is considered as snowfall. The three lines a, b and c in Figure 8 correspond to threshold values $0.5,1.0$ and $2.0 \mathrm{~mm} \mathrm{~h}^{-1}$, respectively. The absolute values of the gradients of the straight lines decrease with increasing threshold values. Therefore, the fractal dimension depends on them. This indicates that the fractal dimension depends on the method used to measure snowfall, because, in general, a threshold value (an accuracy to detect or recognize snowfall) differs according to each method.

Figure 9 shows examples of the $\log$-log plots on the records of daily new-snow depth in Nagaoka. Table 1 summarizes fractal dimensions obtained by these log-log plots of daily new-snow depth, maximum snow depths in Nagaoka and Shinjo in winter (Abe and others, 1985), and total sum of daily new-snow depths.

\section{CONCLUSION}

In this paper, time variation of snowfall and a layered structure of snow cover are interpreted from the viewpoint of fractals, because they have a structure endlessly repeated to a finer structure. In this model, both the total time of snowfall and the number of snow layers depend on scale. Therefore, they cannot be determined absolutely.

It is well known that the shape of clouds is a fractal related to atmospheric turbulence. In the same way, time

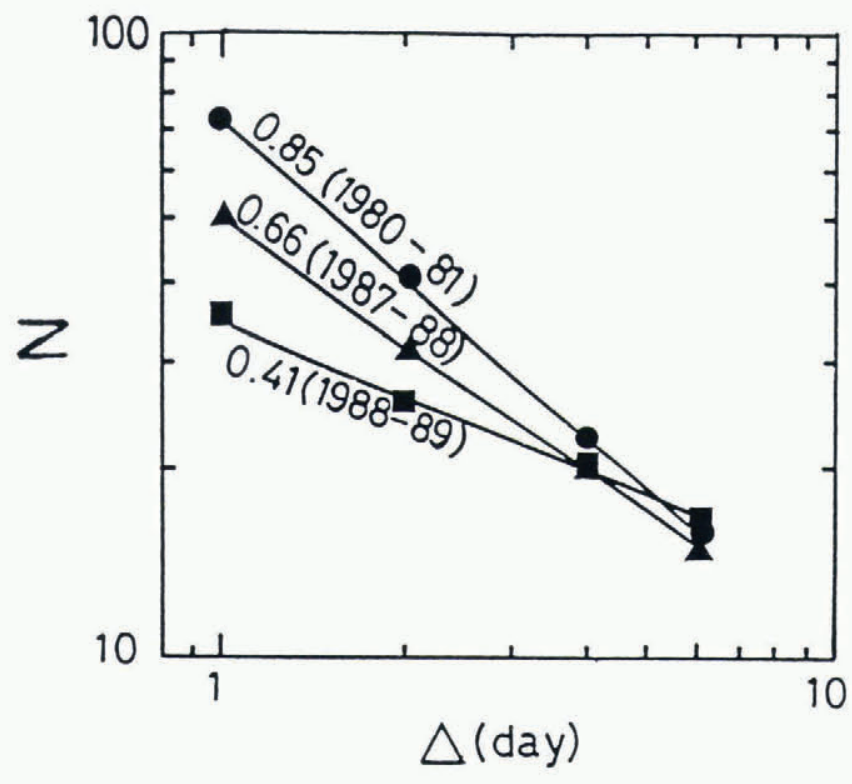

Fig. 9. Log-log plots of daily new-snow depth in Nagaoka, Japan.
Table 1. Fractal dimension, maximum snow depth and total sum of daily new-snow depth in Nagaoka and Shinjo, Japan

\begin{tabular}{|c|c|c|c|c|}
\hline \multirow[t]{2}{*}{ Site } & \multirow[t]{2}{*}{ Year } & \multirow[t]{2}{*}{$\begin{array}{c}\text { Fractal } \\
\text { dimension }\end{array}$} & \multirow{2}{*}{$\begin{array}{c}\text { Maximum } \\
\text { snow depth } \\
\text { m }\end{array}$} & \multirow{2}{*}{$\begin{array}{l}\text { Total sum of } \\
\text { daily new-snow } \\
\text { depth } \\
\text { m }\end{array}$} \\
\hline & & & & \\
\hline \multirow[t]{13}{*}{ Nagaoka } & $1976-77$ & 0.75 & 217 & 894 \\
\hline & $1977-78$ & 0.67 & 123 & 680 \\
\hline & 1978-79 & 0.68 & 74 & 347 \\
\hline & 1979-80 & 0.57 & 240 & 747 \\
\hline & $1980-81$ & 0.85 & 282 & 1052 \\
\hline & $1981-82$ & 0.55 & 117 & 576 \\
\hline & $1982-83$ & 0.58 & 181 & 783 \\
\hline & $1983-84$ & 0.77 & 260 & 1130 \\
\hline & $1984-85$ & 0.70 & 211 & 1070 \\
\hline & $1985-86$ & 0.69 & 254 & 1256 \\
\hline & $1986-87$ & 0.63 & 110 & 716 \\
\hline & $1987-88$ & 0.66 & 145 & 717 \\
\hline & 1988-89 & 0.41 & 63 & 248 \\
\hline \multirow[t]{9}{*}{ Shinjo } & $1974-75$ & 0.78 & 126 & 771 \\
\hline & $1975-76$ & 0.78 & 131 & 612 \\
\hline & $1876-77$ & 0.84 & 146 & 813 \\
\hline & $1977-78$ & 0.81 & 147 & 732 \\
\hline & 1978-79 & 0.65 & 75 & 389 \\
\hline & $1979-80$ & 0.72 & 166 & 766 \\
\hline & $1980-81$ & 0.75 & 183 & 926 \\
\hline & $1981-82$ & 0.70 & 144 & 785 \\
\hline & $1982-83$ & 0.68 & 122 & 653 \\
\hline
\end{tabular}

variation of precipitation also is affected by atmospheric turbulence. Therefore, snow cover, which is a record of snowfall, keeps a measure of the fractal of atmospheric phenomena in its layered structure.

Snowflakes are famous fractals for their shape (Mandelbrot, 1981) and also a letter from heaven (Nakaya, 1938) in a microscopic sense. Similarly, snow cover also is interesting as both a fractal geometry and a record, in a macroscopic sense, of how the heavens inform us of atmospheric-turbulence phenomena.

\section{REFERENCES}

Abe, O., H. Nakamura, M. Higashiura, N. Numano and T. Nakamura. 1985. Snow depth, newly fallen snow depth and weather observed for ten winter seasons at the Shinjo Branch of the NRCDP, Yamagata, Japan (1974/75 winter to 1983/84 winter). Review of Research for Disaster Prevention 106.

Feder, J. 1988. Fractals. New York, Plenum Press.

Kawakami, S. and E. Yoshida. 1988. Fractal and statistical properties of rain-rate processes. Tenki, 35, 693-700. [In Japanese.] 
Lovejoy, S. 1982. Area-perimeter relation for rain and cloud area. Science, 216, 185-187.

Mandelbrot, B. B. 1981. The fractal geometry of nature. New York, W.H. Freeman and Co.

Mandelbrot, B. B. 1986. Self-affine fractal sets. In Pietronero, L. and E. Tossati, eds. Fractals in physics. Amsterdam, North-Holland, 3-28.

Nakaya, U. 1938. Snow. Tokyo, Iwanami. [In Japanese.]
Shimizu, H. 1965. Snow observation method. Tokyo, Japanese Society of Snow and Ice. [In Japanese.]

Wakahama, G. 1963. The infiltration of melt water into snow cover I. Low Temp. Sci., Ser. A 21, 45-73. [In Japanese with English summary.]

The accuracy of references in the text and in this list is the responsibility of the authors, to whom queries should be addressed. 